\title{
Wave localization in random networks
}

\author{
Zhao-Qing Zhang* and Ping Sheng \\ Exxon Research \& Engineering Co., Rt. 22 East, Annandale, New Jersey 08801
}

(Received 12 July 1993)

\begin{abstract}
A random network is defined as a random array of waveguide segments with variable lengths. Each waveguide segment provides a continuous one-dimensional propagation channel. Wave scattering occurs only at the nodes of the network. The network model is shown to be equivalent to a zero-energy state of an equivalent tight-binding Hamiltonian. There can be two kinds of randomness in a network. The coordination number at each node and the length of each waveguide segment can be varied independently. A network becomes ordered only when each node has the same coordination number and all the segments are either equal in length or differ by some integral multiple of the wavelength. A uniformly extended state is found for any random network provided all the segments are of some integral multiple of the wavelength, modulo $2 \pi$. Both analytical and numerical approaches are used to investigate the critical localization behavior in the vicinity of either the uniform state or the ordered network. Our results show that the network model belongs to the same universality class as the disordered tight-binding Anderson model, but with special tunable characteristics. It is thus suggested that a random network of optical fibers can be an effective system for the observation of light localization.
\end{abstract}

\section{INTRODUCTION}

In the past decade, there has been extensive interest on the localization behaviors of both quantum ${ }^{1}$ and classical waves $^{2}$ in random media. Most of these studies were carried out on the tight-binding Anderson model, where the wave-function amplitude is defined only on the nodes of a discrete lattice, and the neighboring nodes are linked by a transition matrix element that can either be a constant or a random variable. In this work, we study the localization behavior of random networks in $1 \mathrm{D}$ and 2D. The network consists of many waveguide segments of variable length joined together at nodes in some random manner. Each segment provides a channel for continuous wave propagation. The scattering occurs only at nodal points where a few segments are joined together. Flux conservation is imposed at each nodal point. There can be two kinds of randomness in a network. The coordination number at each node and the length of each waveguide segment can be varied independently. A network becomes ordered only when each node has the same coordination number and all the segments are either equal in length of differ by some integral multiple of the wave length. The disordered network model is relevant to many physical systems such as propagation of quantum particles in porous media, random optical-fiber network, random network of elastic rod, etc., in which the cross section of each network segment is small so that only one transverse channel is dominant and the cross-channel scattering can be ignored. We show that the network model can be mapped to a zero-energy state of an equivalent tight-binding Hamiltonian (TBH). This mapping first appeared in the study of the phase diagram of superconducting networks under a magnetic field., ${ }^{3,4}$ Here, we are interested in the wave localization properties as a function of wavelength and the disorder parameters.
Our results indicate that even in a network where the coordination number varies from node to node, a uniform state, with constant wave function at every node, appears whenever all the segments are of some integral multiple of the wavelength, modulo $2 \pi$. Various approaches are used to investigate the critical localization behavior in $1 \mathrm{D}$ and $2 \mathrm{D}$. In $1 \mathrm{D}$, the results of a transfer-matrix calculation ${ }^{5}$ shows that the localization length diverges linearly with the mean-free path. In $2 D$, finite-size scaling ${ }^{6}$ results from a recursive Green's function calculation ${ }^{7}$ give a universal function ${ }^{6}$ as found in the Anderson Hamiltonian. These results clearly suggest that the network model belongs to the same universality class as the usual tightbinding disordered model, but with special tunable characteristics. That means not only can one use a random fiber optic array to experimentally test Anderson localization, but its special characteristics may also be of interest, for example, to the management of future optic communication networks.

In what follows, the formulation of a network Hamiltonian and its general properties are discussed in Sec. II. Sections III and IV are devoted to the study of $1 \mathrm{D}$ and $2 \mathrm{D}$ cases, respectively. A brief summary and discussions are given in Sec. $\mathrm{V}$.

\section{FORMULATION}

Consider a network formed by waveguide segments of variable length joining together a random array of nodes. Each segment has as a single-channel for wave propagation. The wave function $\varphi_{i j}$ within any segment between nodes $i$ and $j$ satisfies the following homogeneous wave equation:

$$
\frac{\partial^{2}}{\partial_{s}^{2}} \varphi_{i j}(s)=\frac{\omega^{2}}{c^{2}} \varphi_{i j}(s),
$$


where $\omega$ is the frequency of the wave, $c$ is the wave speed, and $s$ is the distance measured from node $i$. For the Schrödinger equation, one simply replaces the factor $\omega^{2} / c^{2}$ in Eq. (1) by $2 m E / \hbar$. It is known that the solution of Eq. (1) has the form ${ }^{4}$

$$
\varphi_{i j}(s)=\varphi_{i} \frac{\sin \left[\left(l_{i j}-s\right) k\right]}{\sin \left(l_{i j} k\right)}+\varphi_{j} \frac{\sin (s k)}{\sin \left(l_{i j} k\right)},
$$

where $k=\omega / c, l_{i j}$ is the length of the segment, and $\varphi_{i}$ and $\varphi_{j}$ are, respectively, the values of the wave function at the nodes $i$ and $j$. At each node $i$, the wave function is continuous. The derivative of the wave function at the node $i$ gives the following flux conservation condition: ${ }^{4}$

$$
\sum_{j}\left[\frac{\partial}{\partial s} \varphi_{i j}(s)\right]_{s=0}=0,
$$

where the summation $j$ is over all the nodes linked directly to $i$. Substituting Eq. (2) into Eq. (3), we have

$$
-\varphi_{i} \sum_{j} \cot \left(\theta_{i j}\right)+\sum_{j} \varphi_{j} / \sin \left(\theta_{i j}\right)=0,
$$

where $\theta_{i j}=k l_{i j}$. Equation (4) represents the zero-energy state of an equivalent TBH with correlated site energy $\epsilon_{i}=\Sigma_{j} \cot \left(\theta_{i j}\right)$ and hopping matrix $t_{i j}=1 / \sin \left(\theta_{i j}\right)$. Some features of the network model is given below.

First, we note that the wave function at each node $i$ remains unchanged if $\theta_{i j}$ in Eq. (4) is replaced by $\theta_{i j}+2 \pi n_{i j}$ for any integer $n_{i j}$. Physically, this replacement simply reflects a change of length $l_{i j}$ by an amount $n_{i j} \lambda$, which does not affect the wave function at the nodes. Therefore, even though Eq. (4) has a tight-binding form, the spectrum of the wave equation is nevertheless unbounded. In the following, we consider only the values of $\theta_{i j}$ in the interval $[0,2 \pi]$.

In the case of an ordered network, where $\theta_{i j}=\theta$, we show that the density of states (DOS) can be obtained easily from Eq. (4), which now becomes

$$
-\varphi_{i} Z \cos \theta+\sum_{j} \varphi_{j}=0,
$$

where $\theta=l k$, and $l$ is the length of the segment, and $Z$ denotes the coordination number of each node. For an ordered $d$-dimensional hypercubic lattice with nearestneighbor $(n-n)$ interaction, $Z=2 d$. Equation (5) corresponds to an electron with energy $\bar{E}=Z \cos (l \omega / c)$ in a lattice with zero site energy and unit value for the nearest-neighbor hopping matrix element. Thus, the DOS, $\rho(\omega)$, of the network wave equation is the product of the DOS of the TBH, ${ }^{8} \rho_{\mathrm{TBH}}(\bar{E})$, times the factor $d \bar{E} / d \omega$, i.e.,

$$
\rho(\omega)=\rho_{\mathrm{TBH}}(\bar{E}) \frac{d \bar{E}}{d \omega},
$$

with $\bar{E}=Z \cos (l \omega / c)$. Similarly, for the Schrödinger equation, where $\omega^{2} / c^{2}=2 m E / \hbar^{2}$ in Eq. (1), the DOS has the form

$$
\rho(E)=\rho_{\mathrm{TBH}}(\bar{E}) \frac{d \bar{E}}{d E} .
$$

Equations (6a) and (6b) can be easily checked in 1D. Using $^{8}$

$$
\begin{aligned}
& \rho_{\mathrm{TBH}}^{\mathrm{1D}}(\bar{E})=\left(\pi l \sqrt{4-\bar{E}^{2}}\right)^{-1}, \\
& \frac{d \bar{E}}{d \omega}=l \sqrt{4-\bar{E}^{2}} / c,
\end{aligned}
$$

and

$$
d \bar{E} / d E=l \sqrt{\left(4-\bar{E}^{2}\right) m / 2 E} / \hbar,
$$

we recover the correct DOS $\rho(\omega)=(\pi c)^{-1}$ for a continuous 1D classical wave equation and $\rho(E)=\sqrt{m / 2 E} / \pi \hbar$ for the 1D Schrödinger equation.

A very unique feature of the network Schrödinger equation model given by Eq. (5) is the case when $\theta_{i j}=\theta=0$. In this limit, $\varphi_{i}$ has the constant value at every $i$ even when the coordination number at each node $i$ varies randomly, i.e., with $Z$ replaced by $Z_{i}$ in Eq. (5). This case corresponds to a TBH with strong site-bond correlations with the site energy $\epsilon_{i}=-Z_{i}$. The zeroenergy state is then always represented by a constant value, independent of the random configuration. We denote this the uniform state. In the vicinity of a uniform state, the mean-free path, which is the length scale where the phase at the nodes becomes randomized, diverges. It should be noted that the present uniform state is different in nature from the other zero-energy extended state found in a purely off-diagonal disordered $\mathrm{TBH}^{5,9}$ In that case, the wave function is random and has an envelope which behaves as

$$
|\varphi(R)| \sim \exp (-a \sqrt{R})
$$

in $1 \mathrm{D},{ }^{5,9}$ and one can hardly define a mean-free path in the vicinity of this extended state. The interesting questions one can raise here are as follows: (1) How does the localization length diverge as this uniform state is approached, i.e., fixing $Z_{i}$ while letting $\theta_{i j}=\theta \rightarrow 0$; (2) Does this divergent behavior form a new universality class. We will study these questions in detail for 1D and 2D systems in the next two sections.

\section{1D RANDOM NETWORKS}

In $1 \mathrm{D}$, we label the nodes by successive integers. Node 2 and node $N$ at the two ends of the 1D chain are connected to two ordered 1D leads. This is shown in Fig. 1. The network equation (4) becomes

$$
\varphi_{i} \epsilon_{i}+t_{i, i+1} \varphi_{i+1}+t_{i, i-1} \varphi_{i=1}=0,
$$

where

$$
t_{i, i+1}=\sum_{m=1}^{n(i)} \frac{1}{\sin \theta_{i, i+1}^{(m)}}
$$

and

$$
\epsilon_{i}=-\sum_{m=1}^{n(i-1)} \cot \theta_{i-1, i}^{(m)}-\sum_{m=1}^{n(i-1)} \cot \theta_{i, i+1}^{(m)} .
$$

Here $n(i)$ is the number of segments between nodes $i$ and 


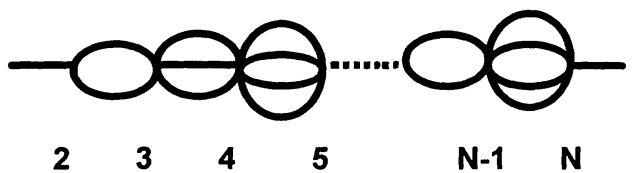

FIG. 1. Schematic picture of a 1D random network.

$i+1$, and $\theta_{i, i+1}^{(m)}=k l_{i, i+}^{(m)}$ is the corresponding phase change in the $m$ th segment. For an ordered 1D network, we have $n(i)=1$ and $\theta_{i, i+1}=\theta$. Let us define $\Delta n$ as the average number of extra segments in the random network, i.e.,

$$
\Delta n=\left\langle\sum_{i=2}^{N-1}[n(i)-1]\right\rangle_{\text {conf }} /(N-2) \text {. }
$$

$\Delta n$ measures the concentration of impurities. In order to have a connected network, we restrict the random integer $n(i)$ to be either greater or equal to 1 . Thus, the value of $\Delta n$ is always positive. The subscript "conf" in Eq. (10) means configurational averaging. Two extended states limits can be obtained by taking (i) $\Delta n \rightarrow 0$ and (ii) $\theta_{i-i, i}^{(m)}=\theta \rightarrow 0$ while $\Delta n \neq 0$. The former is the ordered 1D limit, while the latter case is the uniform state limit, i.e., the wave-function amplitude $\varphi_{i}=$ const.

The localization behavior in the vicinities of these two limits can be studied through the transmission coefficient variation as a function of $N$, the number of nodes along a 1D chain. We use the transfer matrix method ${ }^{5}$ described below to calculate the transmission coefficient. First, Eq. (7) provides the following recursion relation

$$
\begin{aligned}
\left(\begin{array}{c}
\varphi_{i+1} \\
\varphi_{i}
\end{array}\right] & =\left[\begin{array}{cc}
\frac{-\epsilon_{1}}{t_{i, i+1}} & \frac{-t_{i-1, i}}{t_{i, i+1}} \\
1 & 0
\end{array}\right]\left(\begin{array}{c}
\varphi_{i} \\
\varphi_{i-1}
\end{array}\right) \\
& \equiv \tau_{i}\left(\begin{array}{c}
\varphi_{i} \\
\varphi_{i-1}
\end{array}\right] .
\end{aligned}
$$

In the pure lead regions the wave function can be expressed as two traveling waves, i.e.,

$$
\begin{aligned}
& \varphi_{L}=I e^{i k\left(x-x_{0}\right)}+O e^{-i k\left(x-x_{0}\right)}, \\
& \varphi_{R}=I^{\prime} e^{i k\left(x-x_{n+1}\right)}+O^{\prime} e^{-i k\left(x-x_{n+1}\right)},
\end{aligned}
$$

where $x_{0}$ and $x_{n+1}$ are the positions of the zeroth and $(n+1)$ th nodes. $I$ and $I^{\prime}$ are the amplitudes of incoming waves from left and right leads, respectively, while $O$ and $O^{\prime}$ are the corresponding amplitudes of the outgoing waves. These amplitudes are related by transmission and reflection coefficients through the $S$ matrix.

$$
\left[\begin{array}{c}
O \\
O^{\prime}
\end{array}\right]=S\left[\begin{array}{c}
I \\
I^{\prime}
\end{array}\right)=\left(\begin{array}{ll}
r & t^{\prime} \\
t & r^{\prime}
\end{array}\right]\left[\begin{array}{c}
I \\
I^{\prime}
\end{array}\right),
$$

where $r\left(r^{\prime}\right)$ and $t\left(t^{\prime}\right)$ are the reflection and the transmission coefficients from incoming waves $I\left(I^{\prime}\right)$, respectively. Equation (13) can be recast into the following form

$$
\left[\begin{array}{l}
O^{\prime} \\
I^{\prime}
\end{array}\right)=T_{N}\left(\begin{array}{l}
I \\
O
\end{array}\right)=\left(\begin{array}{cc}
t-\frac{r^{\prime} r}{t^{\prime}} & \frac{r^{\prime}}{t^{\prime}} \\
\frac{-r}{t^{\prime}} & \frac{1}{t^{\prime}}
\end{array}\right)\left(\begin{array}{l}
I \\
O
\end{array}\right) .
$$

In the leads, let the phase change between any two adjacent nodes by $\pm \theta$. Then, from Eq. (12), we have

$$
\left(\begin{array}{l}
\varphi_{1} \\
\varphi_{0}
\end{array}\right)=\left(\begin{array}{cc}
e^{i \theta} & e^{-i \theta} \\
1 & 1
\end{array}\right)\left(\begin{array}{l}
I \\
O
\end{array}\right) \equiv Q\left(\begin{array}{l}
I \\
O
\end{array}\right)
$$

and

$$
\left(\begin{array}{l}
\varphi_{N+2} \\
\varphi_{N+1}
\end{array}\right]=Q\left[\begin{array}{l}
O^{\prime} \\
I^{\prime}
\end{array}\right]
$$

Combining Eqs. (11), (14), and (15), we finally obtain

$$
T_{N}=Q^{-1} \tau_{N+1} \tau_{N} \cdots \tau_{2} \tau_{1} Q
$$

Equation (16) is calculated numerically for each configuration, from which one obtains $|r|^{2}$ and $|t|^{2}$ through Eq. (14). The localization length $\xi$ is then related to the configurational average of $\ln |t|$ by

$$
\lim _{N \rightarrow \alpha}\langle\ln \mid t(N)\rangle_{\mathrm{conf}}=-N / \xi \text {. }
$$

It should be noted here that the localization length $\xi$ in this network problem measures only the decay of the wave function as a function of the number of nodes. The physical length does not have much meaning here since the length of any segment may be varied by a multiple of wavelength without altering the wave function at the nodes.

We first examine the localization behavior in the vicinity of the ordered network limit, i.e., case (i) $\Delta n \rightarrow 0$. It should be noted that, in 1D, if there is only one segment between any two adjacent nodes, i.e., $n(i)=1$ for all $i$ or $\Delta n=0$, the network is always ordered, independent of the value of $\theta_{i-1, i}$. This is because there is no scattering in the network. For numerical calculations, the number of segments joining the adjacent nodes are set to be either 1 or 2 with probabilities $P_{1}$ and $P_{2}=1-P_{1}$. Thus, we have $\Delta n=P_{2}$ in the disordered region. If the localization length $\xi$ is to diverge proportional to the mean-free path, we should have $\xi \propto(\Delta n)^{-1}=P_{2}^{-1}$ since $\Delta n$ in Eq. (10) measures the concentration of impurities and is inversely proportional to the mean-free path.

By setting $N=300000$, the average of numerical calculation over 50 configurations are shown in Fig. 2, where we have plotted $\ln (\xi)$ vs $\ln (\Delta n)$ in three different cases: (a) when all the segments give rise to equal phase increments $\theta_{i, i+1}^{(m)}=\theta=\pi / 8$, (b) when $\theta_{i, i+1}^{(m)}=\theta=\pi / 4$, and (c) random phase change $\theta_{i j}$ for each segment with a standard deviation of $2 \pi$. As an illustration, we have also plotted in Fig. 3 the transmission coefficient $t(N)$ as a function of $N$ for a single configuration for the case $\theta_{i, i+1}^{(m)}=\theta=\pi / 16$ and $\Delta n=0.332$. The exponential decay of $t(N)$ gives a localization length $\xi \approx 55.6$ nodes. All three curves in Fig. 2 give an expected slope of $1.00 \pm 0.04$. The fact that curve (a) is higher than (b), which is also higher than (c), is because $\pi / 8$ is closer to 


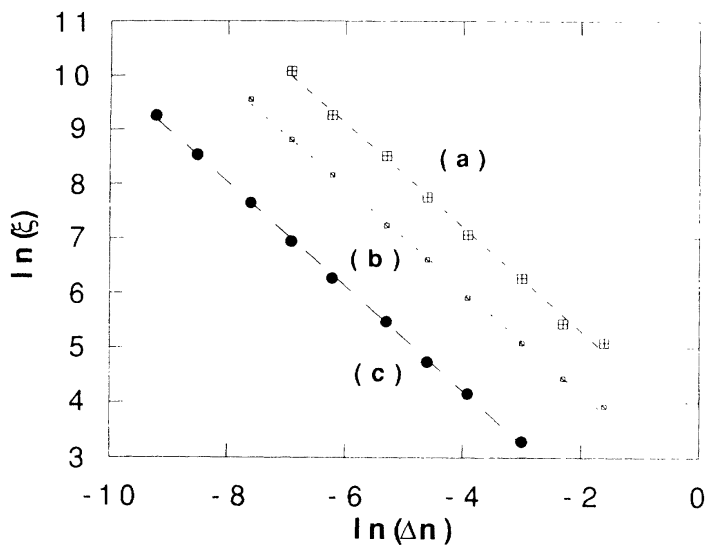

FIG. 2. Log-log plot of the localization length $\xi$ vs the concentration of extra segment in $1 \mathrm{D}$ random networks with (a) $\theta=\pi / 8$, (b) $\theta=\pi / 4$, and (c) random $\theta_{i j}$ of standard deviation $2 \pi$.

the uniform state limit, $\theta=2 \pi n$, than $\pi / 4$.

For the second case, we examine the localization behavior in the vicinity of the uniform state as a function of $\theta$ with $\Delta n \neq 0$. The value of $\xi(\theta)$ is calculated by setting $N=500000$ with 50 configurational average. Figure 4 shows $\ln (\xi)$ vs $\ln (\theta)$ plots for the following two cases: (a) the number of segments between adjacent nodes varies from 1 to 9 with a mean of 4.6, i.e., $\Delta n=3.6$, (b) $n(i)$ varies from 1 to 4 with a mean of 2.2, i.e., $\Delta n=1.2$. The slopes of these two curves are $2.00 \pm 0.06$. The behavior of $\xi(\theta) \propto 1 / \theta^{2}$ is seen in the vicinity of the uniform state. This can be understood by using the following argument. First, in the small $\theta$ limit $\left(\theta \rightarrow 0^{+}\right)$, Eq. (7) can be written as

$$
\begin{aligned}
-\left(n_{i-1, i}+n_{i, i+1}\right) \varphi_{i}+ & n_{i-1, i} \varphi_{i-1}+n_{i, i+1} \varphi_{i+1} \\
& -\left(n_{i-1, i}+n_{i, i+1}\right) \frac{\theta^{2}}{2} \varphi_{i}=0,
\end{aligned}
$$

where $n_{i, i+1}$ is the number of segment between nodes $i$ and $i+1$. Let us compare Eq. (18) with the following

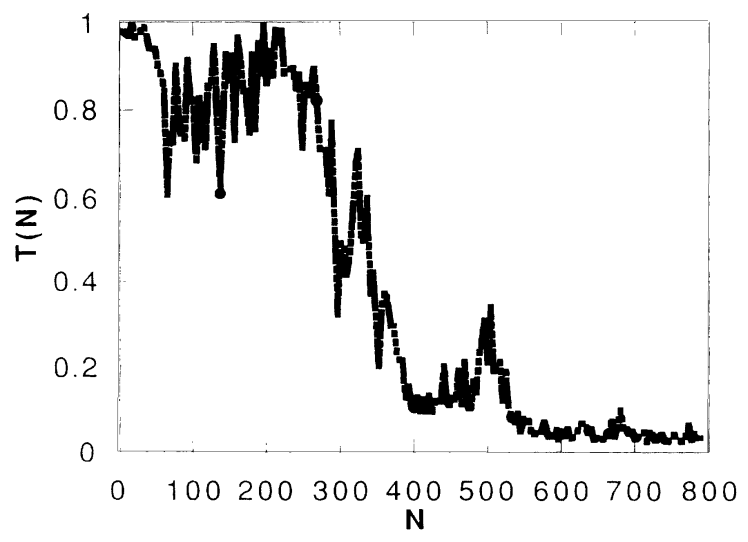

FIG. 3. Single configuration transmission coefficient vs number of nodes in a 1D disordered network with $\theta=\pi / 16$ and $\Delta n=0.332$.

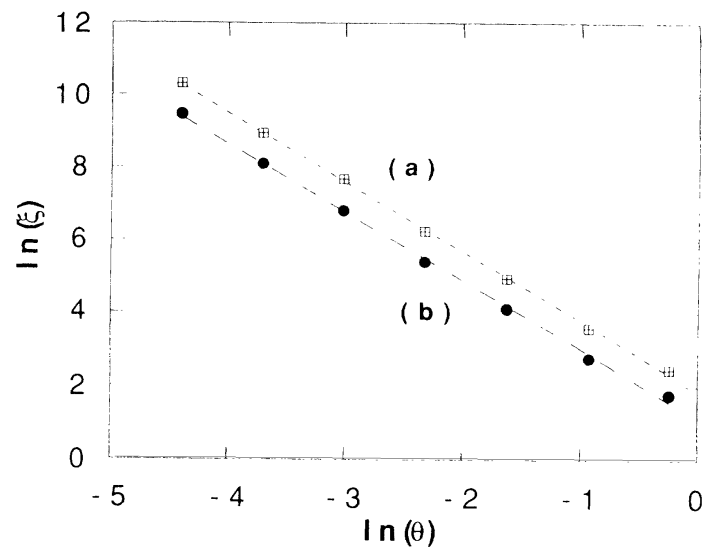

FIG. 4. Log-Log plot of the localization length $\xi$ vs $\theta$ in 1D with (a) $\Delta n=2.2$, (b) $\Delta n=4.6$.

equation of motion for mass-disordered phonon ${ }^{10}$ in $1 \mathrm{D}$ :

$$
-2 U_{i}+U_{i+1}+U_{i-1}+\frac{m_{i} \omega^{2}}{K} U_{i}=O
$$

$\omega$ is the frequency of phonon, where $U_{i}$ is the displacement of site $i, m_{i}$ is the random mass at site $i$, and $K$ is the force constant. When $\theta$ is zero in Eq. (18) and $\omega$ is zero in Eq. (19), both equations give uniform solutions. Thus the uniform state in the disordered network problem can be considered as analogous to the zero frequency Goldstone mode in an ordered system. Both the $\theta^{2}$ and $\omega^{2}$ terms in Eqs. (18) and (19) describe the scattering strength, which randomizes the phases at nodes and cause localization in $1 \mathrm{D}$ and $2 \mathrm{D}$. Thus the behavior of $\xi \propto 1 / \theta^{2}$ is the counterpart of the well-known relation $\xi \propto 1 / \omega^{2}$ in the phonon case. This again indicates that $\xi$ is proportional to a mean-free path that is divergent in the vicinity of a uniform state as $1 / \theta^{2}$.

From the above results, we can conclude that, at least in 1D, this network model falls into the same universality class as the usual disordered TBH, and the $\theta=0$ case (or more generally, $\theta_{i j}=2 \pi n_{i j}$ ) forms the critical surface for any configuration of the network. One can thus use $\theta$ as a tunable parameter to adjust the wave property of a network, such as a fiber-optic array.

\section{2D RANDOM NETWORKS}

In two dimensions, we consider a bond percolation network on a square lattice. The probability that any given segment is absent is $1-p$. The summation $j$ in Eq. (4) is over the occupied segments connecting to a given node $i$. Again, we concentrate our study in both ordered network limit $\left(p \rightarrow 1, \theta_{i j} \neq 0\right)$, and the uniform state limit $(\theta \rightarrow 0, p \neq 1)$. In both cases we first examine the case of constant phase change $\theta_{i j}=\theta$ for all occupied segments. Results of the random phase case will be given subsequently.

The localization behavior in a $2 \mathrm{D}$ network is studied by using the finite-size scaling method. ${ }^{6}$ A strip of percolation network of width $M$ and length $L$ is connected at both ends with two ordered networks as the leads. Since 
the network equation has the tight-binding form, the standard recursive Green's function method ${ }^{7}$ can be used to find the transmission coefficient $t_{M}(L)$ from which one obtains the localization length of the strip $\xi(M)$ as

$$
t_{M}(L)=\text { const } \exp \left(\frac{-2 L}{\xi(M)}\right) \text {. }
$$

In the leads, the network equation is given by Eq. (5) with $Z=4$. Assuming the wave function has the form $\varphi_{i}=e^{i\left(k_{y} y+k_{x} x\right)}$, following relation can be deduced from Eq. (5):

$$
4 \cos \theta-2 \cos k_{y} l-2 \cos k_{x} l=0,
$$

where $l$ is the length of the segment in the lead. In fact, our calculation depends only on the products $k_{y} l$ and $k_{x} l$. If the periodic boundary condition is used in the $y$ direction, we have $k_{y} l=2 m \pi / M$, with $m=0,1,2, \ldots,(M-1)$. The propagating channel is given by the condition that $k_{x} l$ in Eq. (21) is real. The transmission coefficient is given by

$$
t_{M}(L)=\sum_{i=1}^{M_{0}}\left|t_{i j}\right|^{2},
$$

with

$$
\left|t_{i j}\right|^{2}=v_{i} v_{j}\left|G_{i j}^{+}(L+1,0)\right|^{2},
$$

when $M_{0}$ is the number of propagating channels, $v_{i}=-\sin \left(k_{x} l\right) / \sin \theta$, and $G_{i j}^{+}(L+1,0)$ is the retarded Green's function with source at 0 of the $x$ coordinate of channel $j$ and receiver at $L+1$ of the $x$ coordinate of channel $i . G_{i j}^{+}$is calculated by the recursive Green'sfunction method. ${ }^{7}$ Thus, for each configuration one can obtain a localization length $\xi(M)$ from Eq. (20). For configurational average, we use the geometric mean of $\mid \xi(M)]^{-1}$. Since all states are localized except those on the critical surface $\theta=0$, the localization length $\xi$ of the bulk network is approached as $M \rightarrow \infty .{ }^{11}$

Numerically, we have chosen $M=8,16,32,64$, and 128. The choice of the length $L$ and the number of configurations depends on the value of $\xi(M)$. The maximum value of $L$ and the configuration number are 20000 and 40 , respectively. Technically, in order to avoid the numerical singularity when the network breaks up into pieces as $p$ approaches the percolation threshold, $p_{c}=0.5$, we have introduced a nonzero hopping term, $\eta / \sin \left(\theta_{i j}\right)$, in Eq. (4) for any unoccupied segment. ${ }^{11}$ The value of $\eta$ is chosen to be $10^{-5}$. The results of our calculation remain unchanged when $\eta$ is reduced to $10^{-6}$.

In the ordered network limit of $p \rightarrow 1, \xi$ is calculated for $0.55 \leq p \leq 0.85$ with fixed $\theta_{i j}=\theta=\pi / 4$ and $\pi / 3$. For both values of $\theta$, we find that $\xi(p)$ is best fitted by the form

$$
\xi(p)=a\left(\frac{p}{1-p}\right)^{1 / 2} \exp \left[b\left(\frac{p}{1-p}\right]^{1 / 2}\right]
$$

where $a$ and $b$ are independent of $p$. Equation (24) provides a linear relation between

$$
\left(\frac{1-p}{p}\right)^{1 / 2} \ln \left[\left(\frac{1-p}{p}\right)^{1 / 2} \xi(p)\right]
$$

and $\sqrt{(1-p) / p}$. This is seen in Fig. 5. Except for the prefactor, the effect of which is minor, Eq. (24) is consistent with the form obtained in the quantum percolation model. ${ }^{11}$ The accuracy of our calculation in $\xi(p)$ varies from $4 \%$ for $p=0.55$ to $30 \%$ for $p=0.85$ and $\theta=\pi / 4$. The inaccuracy is due to large fluctuations when $\xi(M)$ becomes large. We need at least one order of magnitude increase of computing time in order to reduce the uncertainty significantly at $p=0.85$.

In the uniform-state limit, we have calculated $\xi$ as a function of $\theta$ in the range of $\pi / 128 \leq \theta \leq \pi / 3$, of $p=0.55$ and 0.65 . The best fit is of the form

$$
\xi(\theta)=\frac{a}{\theta} \exp \left(\frac{b}{\theta}\right),
$$

where $a$ and $b$ are independent of $\theta$. The corresponding plot is shown in Fig. 6. Although Eqs. (24) and (25) are consistent with the results of quantum percolation mod$\mathrm{el},{ }^{11}$ we believe that the correct form for $\xi$ should be

$$
\xi(p, \theta)=\frac{a}{\theta^{2}}\left[\frac{p}{1-p}\right] \exp \left[\frac{b p}{\theta^{2}(1-p)}\right),
$$

where $a$ and $b$ are independent of $\theta$ and $p$. Equation (26) falls into the same universality class of the usual Anderson TBH in 2D. As can be seen, the mean-free path $l$ is inversely proportional to $\theta^{2}(1-p)$. In fact, a selfconsistent mode-coupling theory ${ }^{12}$ has predicted that the factor in the exponent of Eq. (24) is $p /(1-p)$ instead of $[p / 1-p]^{1 / 2}$ for the quantum percolation model. If Eq. (26) is correct, we would expect a linear relation between

$$
\frac{(1-p) \theta^{2}}{p} \ln \left[\frac{(1-p) \theta^{2}}{p} \xi(p, q)\right]
$$

and $(1-p) \theta^{2} / p$. However, using our data we find that the deviation from a linear relation is quite severe in both

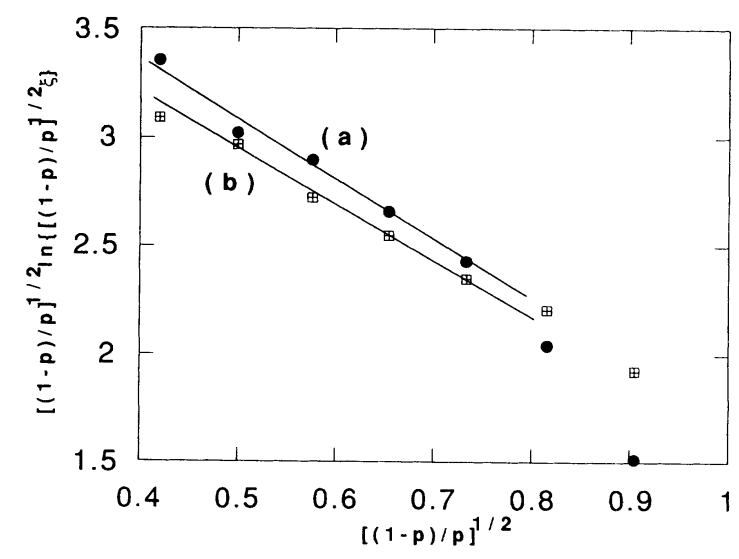

FIG. 5. $[(1-p) / p]^{1 / 2} \ln \{[(1-p) / p] \xi\}$ vs $[(1-p) / p]^{1 / 2}$ for 2D percolation networks with (a) $\theta=\pi / 4$ and (b) $\pi / 3$. The localization $\xi$ is given by Eq. (24) in the text. 


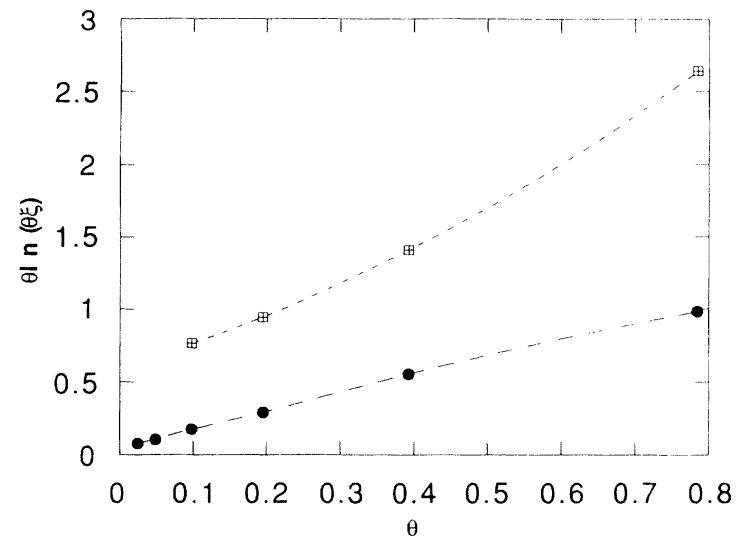

FIG. 6. $\theta \ln (\theta \xi)$ vs $\theta$ for $2 \mathrm{D}$ percolation network with (a) $p=0.65$ and (b) $p=0.55$. The localization length is given by Eq. (25) in the text.

ordered network and uniform-state limits. To resolve the discrepancy, a much more accurate numerical calculation is required, particularly if $p$ is close to 1 so that $\xi$ is large. This has not been done so far.

We have also calculated $\xi$ for the case of random $\theta_{i j}$ with a $2 \pi$ standard deviation. The results of finite-size scaling are shown in Fig. 7 from $p=0.6$ to $1 . \xi(p)$ is now a monotonic function of $p$ and is finite at $p=1$, where Eq. (4) corresponds to the zero-energy state of a correlated site-bond disordered TBH.

The question about the universality class of the network model may be studied by using the following scaling test. According to the finite-size scaling theory, ${ }^{6,11}$ if the network model belongs to the same universality as the usual disordered TBH, we would expect that $\xi(M) / M$ is a function of a single variable $\xi / M$ alone and is independent of all other parameters in the network. Also, this scaling function should be universal, i.e., independent of the details of the disordered Hamiltonian in the large $M$ and $\xi(M)$ limits. To test the scaling and universality, we have generated all the data points from previous results of $\xi(M), M=32,64$, and 128 , and plotted them on a sin-

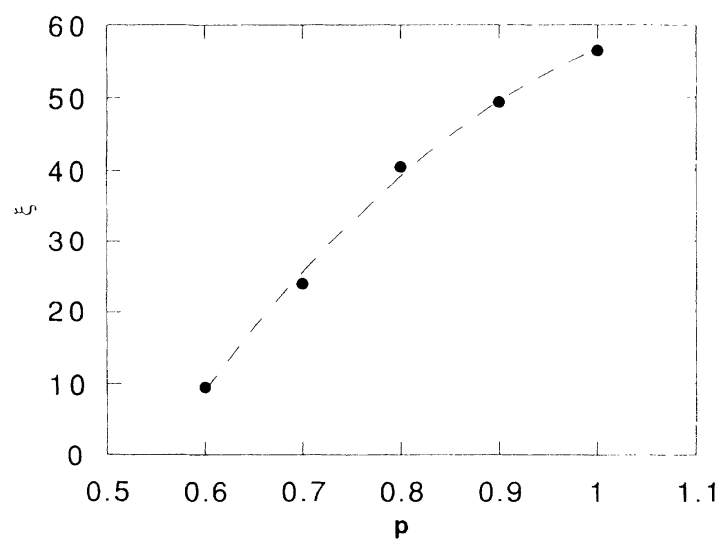

FIG. 7. Localization length $\xi$ vs $p$ in a $2 D$ percolation network with random $\theta_{i j}$ for each segment. The standard deviation of $\theta_{i j}$ is $2 \pi$.

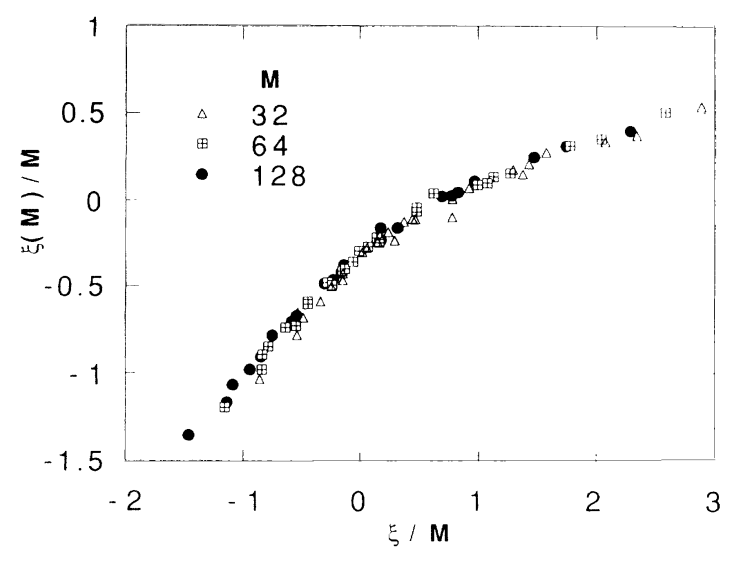

FIG. 8. $\xi(M) / M$ vs $\xi / M$ for $M=32,64$, and 128 with data from three different cases: (a) $0.55 \leq p \leq 0.85, \theta=\pi / 4$, and $\pi / 3$, (b) $\pi / 128 \leq \theta \leq \pi / 3, p=0.55$ and 0.65 , and (c) random $\theta_{i j}$ of variance $2 \pi$. Coincidence of the data indicates universal scaling behavior.

gle figure. In Fig. 8 , it is seen that for the three cases studied, i.e., (a) $0.55 \leq p \leq 0.85, \theta=\pi / 4$, and $\pi / 3$, (b) $\pi / 128 \leq \theta \leq \pi / 3, p=0.55$, and 0.65 , and (c) random $\theta_{i j}$, $\xi(M) / M$ indeed forms a single function of the scaling variable $\xi / M$. This scaling function coincides exactly with the scaling function obtained from either the Anderson Hamiltonian ${ }^{6}$ or the quantum percolation model. ${ }^{11}$ These scaling results indicate that our network model falls in the same universality class as the usual Anderson model.

\section{CONCLUDING REMARKS}

We have studied the wave localization properties of $1 \mathrm{D}$ and $2 \mathrm{D}$ random networks made of segments of singlechannel waveguide jointed in a random array. Our network model is shown to be isomorphic to the zero-energy state of a disordered correlated site-bond TBH. A new uniform state is found when the phase change in each segment is an integer of $2 \pi$, i.e., $\theta_{i j}=2 \pi n_{i j}$. This uniform state is analogous to a Goldstone mode in an ordered system and its existence is independent of the random configuration. Besides the randomness parameters $\Delta n$ and $p$, additional phase parameter $\theta, \theta_{i j}=2 \pi n_{i j}+\theta$, is shown to control the localization length in the network. In both the uniform state limit and the ordered network limit ( $\Delta n=0$ in $1 \mathrm{D}$ or $p=1$ in 2D), our results indicate that the critical behavior of localization falls into the same universality class as the usual Anderson TBH. This equivalence suggests that the network model can be used to observed light localization, i.e., in a fiber-optic array. The tunable parameter $\theta$ could be relevant to actual optical communication networks of the future.

It should be noted that the network model considered here can be easily generalized to the case where each waveguide segment has a different wave speed. In this case, the constants $c$ and $k$ in Eqs. (1) and (2) should be 
replaced by $c_{i j}$ and $k_{i j}$, respectively, and Eq. (4) becomes

$$
-\varphi_{i} \sum_{j} k_{i j} \cot \left(\theta_{i j}\right)+\sum_{j} \varphi_{j} k_{i j} / \sin \left(\theta_{i j}\right)=0,
$$

where $k_{i j}=\omega / c_{i j}$ and $\theta_{i j}=k_{i j} l_{i j}$.

Even in this case, there are uniform states as long as all the segments are of some integral multiple of their respective wavelength, i.e., $k_{i j} l_{i j}=$ integer of $2 \pi$. The system will still belong to the same universality class discussed before. This is certainly true in the following 1D case. Consider a 1D network with only one segment between any two adjacent nodes. If each segment has a different length and wave speed, the system is equivalent to a random dielectric multilayer. It can be shown easily that Eq. (27) describes a random dielectric multilayer system. The localization behavior in such a system has been studied and shown to be in the same universality class as the Anderson TBH. ${ }^{13}$ Many properties of such a system are also known. ${ }^{14}$

Although we did not study the statistical distribution of the transmission coefficient of the network model, it is our belief that the distribution should be log-normal in both 1D and 2D networks. In 1D, there are both analytic and numerical works on the Anderson model with diagonal and/or off-diagonal randomness. ${ }^{5,15-17}$ All results converge to a log-normal distribution. In $2 \mathrm{D}$, this log-normal distribution has also been clearly demonstrated in numerical simulations on a percolation model. ${ }^{18}$ Since our calculations have indicated that the network model belongs to the same universality class as the Anderson TBH, we believe strongly that the statistical distribution of transmission coefficient should also follow a log-normal distribution in both $1 \mathrm{D}$ and 2D.
${ }^{*}$ Also at Institute of Physics, Academia Sinica, Beijing, China.

${ }^{1}$ See, for example, P. A. Lee and T. V. Ramakrishnan, Rev. Mod. Phys. 57, 287 (1985).

${ }^{2}$ See, for example, Scattering and Localization of Classical Waves in Random Media, edited by P. Sheng (World Scientific, Singapore, 1989).

${ }^{3}$ P. G. de Gennes, C. R. Acad. Sci. Ser. B 292, 279 (1981).

${ }^{4}$ S. Alexander, Phys. Rev. B 27, 1591 (1983).

${ }^{5}$ A. Douglas Stone, J. D. Joannopoulos, and D. J. Chadi, Phys. Rev. B 24, 5583 (1981).

${ }^{6}$ A. Mackinnon and B. Kramer, Phys. Rev. Lett. 47, 1546 (1981).

${ }^{7}$ P. A. Lee and D. S. Fisher, Phys. Rev. Lett. 47, 882 (1981).

${ }^{8}$ E. N. Economou, Green's Function in Quantum Physics (Springer-Verlag, Berlin, 1979).
${ }^{9}$ C. M. Soukoulis and E. N. Economou, Phys. Rev. B 24, 5698 (1981).

${ }^{10}$ Q. J. Chu and Z. Q. Zhang, Phys. Rev. B 39, 7120 (1989).

${ }^{11}$ C. M. Soukoulis and G. S. Grest, Phys. Rev. B 44, 4685 (1991).

${ }^{12}$ R. F. Loring and S. Mukamel, Phys. Rev. B 33, 7708 (1986).

${ }^{13}$ P. Sheng, B. White, Z. Q. Zhang, and G. Papanicolaou, Phys. Rev. B 34, 4757 (1988).

${ }^{14}$ Bart A. van Tiggelen, Ph.D. thesis, Universiteit van Amsterdam, Amsterdam, The Netherlands, 1990 and references therein.

${ }^{15}$ A. D. Stone and J. D. Joannopoulos, Phys. Rev. B 24, 3529 (1981).

${ }^{16}$ E. Abrahams and M. J. Stephen, J. Phys. C 13, L377 (1980).

${ }^{17}$ B. S. Andereck and E. Abrahams, J. Phys. C 13, L383 (1981).

${ }^{18}$ Z. Q. Zhang, and P. Sheng, Phys. Rev. B 44, 3304 (1991). 